Ophthalmologe 2021 · 118:512-516 https://doi.org/10.1007/s00347-021-01360-1 Angenommen: 18. Februar 2021 Online publiziert: 19. März 2021 ๑ Der/die Autor(en) 2021

\section{Erprobungsregelung des Ge- meinsamen Bundesausschusses}

Bei der Bewertung, ob medizinische Untersuchungs- und Behandlungsmethoden durch die Krankenkassen erstattet werden, hat der Gemeinsame Bundesausschuss (G-BA) jeweils den aktuellen Stand der medizinischen Erkenntnisse und wissenschaftlichen Datenlage zu berücksichtigen.

Im Fall der transkornealen Elektrostimulation (TES) ist die Studienlage aktuell für eine abschließende Beurteilung des Nutzens dieser Methode noch nicht ausreichend. Die bisherigen Ergebnisse präklinischer und klinischer Studien weisen darauf hin, dass die TES das Potenzial einer nutzenbringenden Behandlungsmethode hat [5-8]. Daher soll diese Methode im Rahmen einer Erprobungsregelung als Studie getestet werden. Mit der Gesamtkoordination des Projekts wurde das Universitätsklinikum Tübingen (UKT) beauftragt, als Partner des UKT

Die Mitglieder der TES-RP-Studiengruppe werden am Beitragsende gelistet.

Nadine Kahle ${ }^{1}$ Tobias Peters ${ }^{1} \cdot$ Angelika Braun$^{1} \cdot$ Jeremy Franklin² •

Claudia Michalik $^{3}$. Florian Gekeler ${ }^{1,4}$ - Barbara Wilhelm ${ }^{5}$ - retina.net e. V. • TES-RPStudiengruppe

' Department für Augenheilkunde, Universitätsklinikum Tübingen, Tübingen, Deutschland

${ }^{2}$ Institut für Medizinische Statistik und Bioinformatik, Medizinische Fakultät, Universität zu Köln, Köln, Deutschland

${ }^{3}$ Zentrum für Klinische Studien, Medizinische Fakultät, Universität zu Köln, Köln, Deutschland

${ }^{4}$ Klinikum der Landeshauptstadt Stuttgart gKAöR, Stuttgart, Deutschland

${ }^{5}$ Steinbeis Transfer Zentrum (STZ) eyetrial am Department für Augenheilkunde, Universitätsklinikum

Tübingen, Tübingen, Deutschland

\title{
Transkorneale Elektrostimulation bei Retinitis pigmentosa
}

\section{Prüfplan einer multizentrischen, prospektiven, randomisierten, kontrollierten und doppelblinden Studie im Auftrag des Gemeinsamen Bundesausschusses (G-BA-Erprobungsrichtlinie)}

sind retina.net e. V., das Steinbeis Transfer Zentrum (STZ) eyetrial am Department für Augenheilkunde des Universitätsklinikums Tübingen und das Zentrum für klinische Studien (ZKS) der Universität zu Köln beteiligt.

Im Folgenden werden die Hintergründe sowie der für die Erprobungsregelung entwickelte Prüfplan zur geplanten Studie, die im Frühjahr 2021 beginnen wird, vorgestellt.

\section{Transkorneale Elektro- stimulation}

\section{Durchführung}

Das OkuStim ${ }^{\circledR}$-System (Okuvision $\mathrm{GmbH}$, Reutlingen), mit dem die transkorneale Elektrostimulation(TES) durchgeführt wird, ist ein zugelassenes und CE-gekennzeichnetes Medizinprodukt, das seit mehreren Jahren von Patienten mit Retinitis pigmentosa (RP) angewendet wird. Bisher erfolgte die Therapie auf Kosten des Patienten oder in Einzelfall-Entscheidungen finanziert durch die Krankenkassen.
OkuStim $^{\circledR}$ besteht aus 3 Komponenten: $\mathrm{OkuStim}^{\circledR}$-Handgerät, OkuSpex-Brille $^{\circledR}$ und $\mathrm{OkuEl}^{\circledR}$-Elektroden (- Abb. 1). Die OkuSpex ${ }^{\circledR}$-Brille dient als Halterung für die $\mathrm{OkuEl}^{\circledR}$-Elektroden und kann individuell an die Gesichtsform des Patienten angepasst werden. Die OkuEl ${ }^{\circledR}$-Elektroden sind so gestaltet, dass sie nur die Bindehaut berühren und so die elektrischen Impulse auf die Augen übertragen können. Im Schläfenbereich wird die Gegenelektrode angebracht. Über die Brille werden die Elektroden stets an beiden Augen angelegt. Den Patienten wird nicht mitgeteilt, welches Auge stimuliert wird und als Studienauge gilt. Bei der Sham-Prozedur wird nach einem initial beidseitigen Stimulationsreiz eine Seite (Auge) ,, abgeschaltet“ und somit nicht mehr stimuliert. Der Patient hat so den Eindruck einer Stimulation beider Augen bzw. kann nicht zuordnen, welches Auge behandelt wird (Verblindung). 


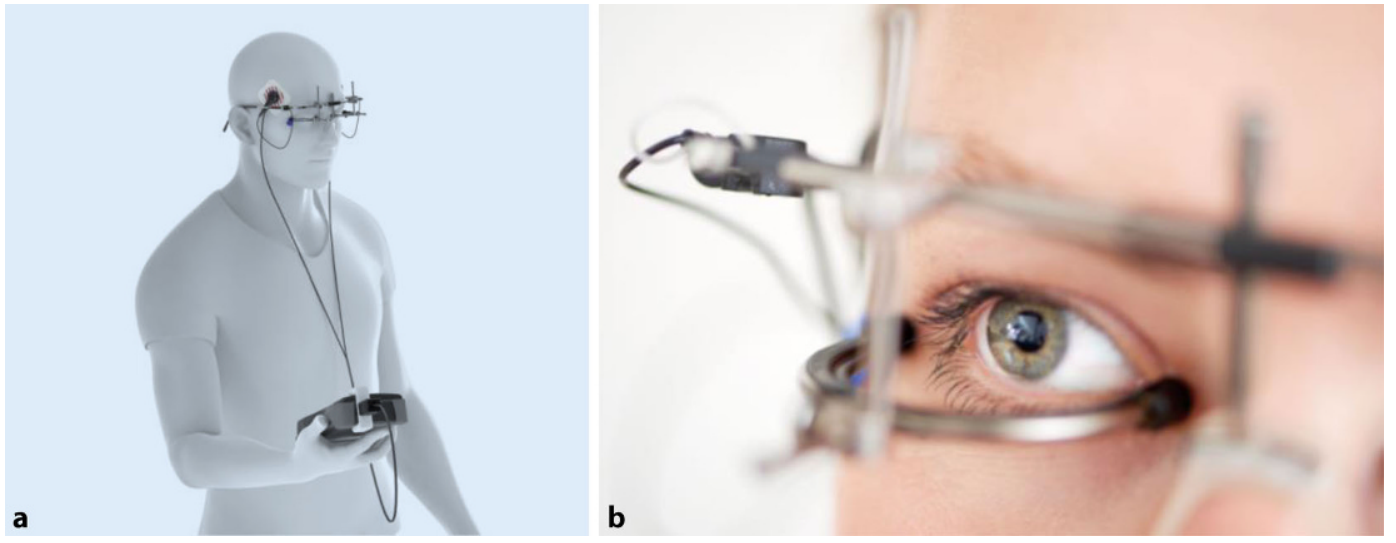

Abb. $1<$ a OkuStim ${ }^{\circledR}$-System (Fa. Okuvision $\mathrm{GmbH}$, Reutlingen): OkuSpex ${ }^{\circledR}$ Brille mit OkuEl ${ }^{\circledR}$-Elektroden und Handgerät. $\mathbf{b}$ Stimulation: Berührung der Bindehaut des Auges durch die mit der OkuSpex ${ }^{\circledR}$-Brille angebrachte $\mathrm{OkuEl}^{\circledR}$-Elektrode, Übertragung elektrischer Impulse auf das Auge über die Elektrode. (Mit freundl. Genehmigung der Fa. Okuvision $\mathrm{GmbH}$, Reutlingen)

\section{Wirkung}

Vorangegangene präklinische und klinische Studien zeigten positive Trends und teils signifikante Ergebnisse, die darauf hinweisen, dass die TES ein vielversprechender Therapieansatz für Patienten mit RP sein kann [6-8]. Die Wirkungsweise der Elektrostimulation wurde dabei bisher auf folgende Mechanismen, oder eine Kombination aus mehreren dieser Mechanismen, zurückgeführt [10]: Regulation neurotropher [6], vasodilatatorischer $[1,2,4]$ sowie antiapoptotischer und antientzündlicher Faktoren und Mechanismen [11]. Um diese Effekte aufrechtzuerhalten, scheint eine dauerhafte Therapie notwendig zu sein [9].

Während der Durchführung der bisher veröffentlichten klinischen Studien traten keine schweren Nebenwirkungen auf. Häufige, vorübergehende Beschwerden sind einfach zu behandelnde trockene Augen.

Insgesamt kommen die bisherigen Studien zu dem Schluss, dass größere Patientenzahlen und längere Beobachtungszeiten erforderlich sind, um ergänzende, belastbare Daten zu erhalten und die positiven Effekte der Elektrostimulation, also die Verlangsamung des Krankheitsverlaufs, gemessen am Gesichtsfeld, bei RP-Patienten zeigen zu können. Diese Evidenzlücke soll mit der geplanten Studie geschlossen werden.

\section{Indikation}

Retinitis pigmentosa (RP) bezeichnet eine Familie erblicher Netzhautdystrophien mit einer durchschnittlichen
Prävalenz von 2,2/10.000 in Europa. Sie ist gekennzeichnet durch eine fortschreitende Netzhautdegeneration, die in der Peripherie beginnt und sich allmählich zum Zentrum der Netzhaut hin ausdehnt, wodurch sich das Gesichtsfeld im Krankheitsverlauf stetig verringert. In - Abb. 2 sind die Unterschiede zwischen Gesichtsfeldmessung und Fundusfoto eines gesunden Probanden ( $\mathbf{A} \mathbf{A b b} \mathbf{2} \mathbf{2}, \mathbf{b})$ im Vergleich zu jenen eines RP-Patienten (- Abb. 2c, d) dargestellt.

Beginnend oft mit Nachtblindheit im ersten oder zweiten Lebensjahrzehnt, kommt es meist im dritten und vierten Lebensjahrzehnt zum Verlust des peripheren Sehens (Tunnelblick) bis hin zur vollständigen Erblindung im Spätstadium. Die RP stellt eine der häufigsten Ursachen für Blindheit oder schwere Sehschwäche bei Menschen im Alter von 20 bis 60 Jahren dar [3].

Dass die Patienten aufgrund des fortschreitenden Verlaufs der Krankheit Schritt für Schritt ihr Sehvermögen verlieren und es derzeit noch - mit Ausnahme der Gentherapie einer einzigen Mutation - keine zugelassene Therapie gibt, die den Krankheitsverlauf verlangsamen oder stoppen kann, ist sowohl für die Betroffenen als auch für die behandelnden Ärzte eine große Herausforderung und Belastung. Deshalb ist es von großer Relevanz, effektive Behandlungsmethoden für diese zur Blindheit führende Krankheit zu entwickeln.

\section{Kriterien für die Studien- teilnahme}

\section{Einschlusskriterien}

1. Patienten mit syndromaler oder nichtsyndromaler RP (autosomaldominant, autosomal-rezessiv und „x-linked“),

2. Erwachsene jeden Geschlechts im Alter von 18-80 Jahren,

3. bestkorrigierte Sehschärfe (ETDRSVisus, gemäß Early Treatment Diabetic Retinopathy Study) zwischen 0,1 und 0,8 in beiden Augen mit nicht mehr als 15 ETDRS-Buchstaben (3 Zeilen) Unterschied zwischen den Augen,

4. Octopus 900 (Fa. Haag-Streit, Wedel), kinetisches Gesichtsfeld:

a. Gesichtsfeldfläche $\geq 75 \mathrm{deg}^{2}$ (entspricht einem durchschnittlichen Radius von 5 Grad; Stimulus V4e) in beiden Augen,

b. der Unterschied zwischen beiden Augen darf eine Ratio „OD/OS“ (Oculus dexter/Oculus sinister) von nicht unter 0,5 und nicht mehr als 2 aufweisen,

5. Patienten in der Lage und willens, das schriftliche Einverständnis zur Studie zu geben.

\section{Wichtigste ophthalmologische Ausschlusskriterien}

1. Optikusneuritis/Optikusneuropathie,

2. diabetische Retinopathie,

3. Neovaskularisationen der Netzhaut unterschiedlicher Genese, 
4. Zustand nach retinalem Arterienoder Venenverschluss,

5. Zustand nach Netzhautablösung oder nach vitreoretinaler Chirurgie,

6. Silikonöltamponade,

7. trockene oder neovaskuläre altersbedingte Makuladegeneration (AMD),

8. Makulaödem,

9. Glaukom,

10. Hornhautdegeneration mit Auswirkung auf die Sehschärfe,

11. Trübung der brechenden Medien, die eine optische Kohärenztomographie (OCT)/Ophthalmoskopie beeinträchtigen,

12. Katarakt, die nach Einschätzung des Prüfers im Studienzeitraum operationsbedürftig werden kann,

13. Aphakie,

14. TES-Therapie in den letzten 3 Monaten (Teilnahme ist nach einer 3-monatigen Therapiepause möglich).

\section{Studienablauf und Unter- suchungen}

Ab Frühjahr 2021 werden deutschlandweit 154 Patienten an 18 deutschen Studienzentren rekrutiert. Pro Patient beträgt die Studiendauer 3 Jahre und umfasst insgesamt 8 Termine, zu denen die Patienten an die Prüfzentren kommen. In einer Screening-Visite werden zunächst alle Ein- und Ausschlusskriterien überprüft.

Jeder Patient und seine Angehörigen erhalten zu Beginn der Studie an der Klinik eine genaue Einweisung in die Anwendung und Handhabung des OkuStim $^{\circledR}$-Systems, um dieses während der Studie selbstständig und studienkonform zu Hause selbst anwenden zu können.

Die Stimulationsdauer beträgt $30 \mathrm{~min}$ einmal wöchentlich über einen Zeitraum von 3 Jahren. Die Stimulationsstärke ist auf $0,8 \mathrm{~mA}$ festgelegt und wird durch das Handgerät mit biphasischen Pulsen und einer Pulsfrequenz von $20 \mathrm{~Hz}$ bereitgestellt. Sollten bei der Behandlung mit 0,8 mA Missempfindungen auftreten, kann die Stimulationsstärke auf $0,6 \mathrm{~mA}$ reduziert werden.

Ophthalmologe $2021 \cdot 118: 512-516$ https://doi.org/10.1007/s00347-021-01360-1

(c) Der/die Autor(en) 2021

N. Kahle - T. Peters · A. Braun - J. Franklin · C. Michalik · F. Gekeler • B. Wilhelm • retina.net e. V. TES-RP-Studiengruppe

Transkorneale Elektrostimulation bei Retinitis pigmentosa. Prüfplan einer multizentrischen, prospektiven, randomisierten, kontrollierten und doppelblinden Studie im Auftrag des Gemeinsamen Bundesausschusses (G-BA-Erprobungsrichtlinie)

\section{Zusammenfassung}

Der Schwerpunkt dieser großen, multizentrischen Erprobungsstudie im Auftrag des Gemeinsamen Bundesausschusses (G$B A)$ liegt in der Feststellung eines Nutzens der transkornealen Elektrostimulation für Patienten mit Retinitis pigmentosa (RP). Hauptkriterium ist das kinetische Gesichtsfeld und die Frage, ob die Verschlechterung der Studienaugen im Vergleich zu den zum Schein (sham-)stimulierten Partneraugen über einen Behandlungszeitraum von 3 Jahren langsamer fortschreitet.

Schlüsselwörter

Retinadystrophien · Retinadegeneration . Netzhauterkrankungen · Elektrische Stimulationstherapie. Gesichtsfeld

Transcorneal electrostimulation in retinitis pigmentosa. Protocol of a multicentric prospective, randomized, controlled and doublemasked trial on behalf of the Joint Federal Committee (G-BA pilot regulation)

Abstract

The focus of this large multicenter trial commissioned by the Joint Federal Committee (Gemeinsamer Bundesausschuss, G-BA) is to determine a benefit of transcorneal electrical stimulation for retinitis pigmentosa (RP) patients. The main criterion for benefit is the kinetic visual field and whether the deterioration progresses more slowly in the study eyes compared to the sham-stimulated fellow eyes over a treatment period of 3 years.

\section{Keywords}

Retinal dystrophies - Retinal degeneration . Retinal diseases - Electric stimulation therapy . Visual field
Im Rahmen der Studie wird nur ein zufällig ausgewähltes Auge stimuliert, das Partnerauge dient als Kontrolle und erhält eine Scheinbehandlung. Weder dem Patienten noch dem Studienpersonal inklusive der Ärzte ist bekannt, welches Auge stimuliert wird (randomisiertes, doppelblindes Studiendesign).

Um eine Verminderung des relativen Gesichtsfeldverlusts im stimulierten Auge, verglichen mit dem relativen Gesichtsfeldverlust im zum Schein (sham-)behandelten Auge, bewerten zu können, werden kinetische Gesichtsfelduntersuchungen mit dem Octopus 900 Pro (Fa. Haag-Streit, Wedel) zu 4 Zeitpunkten durchgeführt. Dabei dienen die beiden bei der Screening-Visite durchgeführten Gesichtsfelduntersuchungen der Minimierung von Lerneffekten. Sie finden in der statistischen Analyse des primären Endpunkts keine Verwendung. Weitere Untersuchungsmethoden sind neben der augenärztlichen Routineuntersuchung die spektrale optische Kohärenztomographie („spectral-domain optical coherence tomography“, SDOCT), die Kontrastempfindlichkeit (Pelli-Robson-Tafel), die dunkeladaptierte Empfindlichkeitsschwelle („full-field stimulus testing", FST) und der Roth-28Hue-Test sowie die Befragung anhand des Visual Function Questionnaire 25 (VFQ-25). Alle Studiendaten werden in einer elektronischen Datenbank (elektronischer Prüfbogen, ,electronical case report form“, eCRF) gesammelt und am Studienende statistisch ausgewertet.

Das Sicherheitskomitee (Data Safety Monitoring Board; DSMB) setzt sich aus 


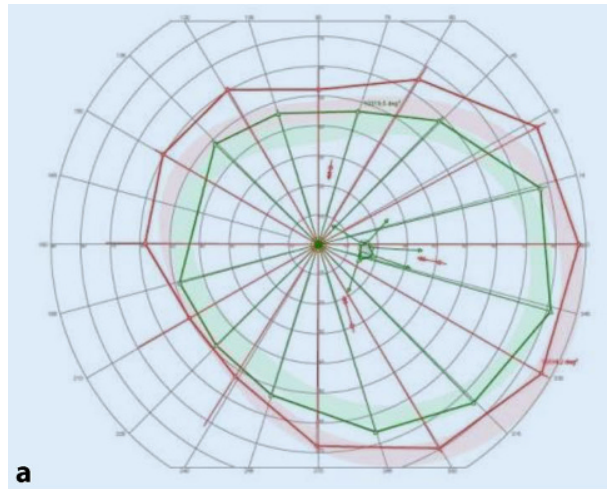

a

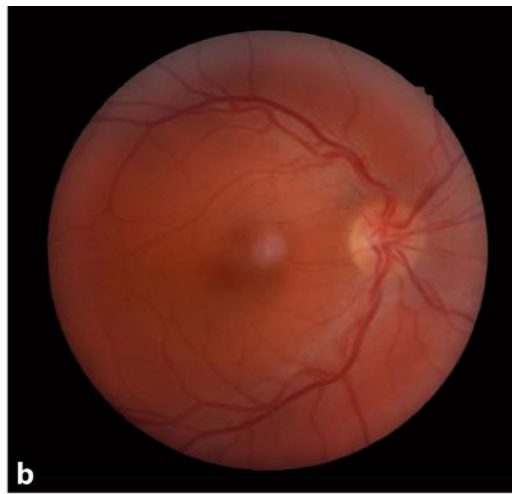

C
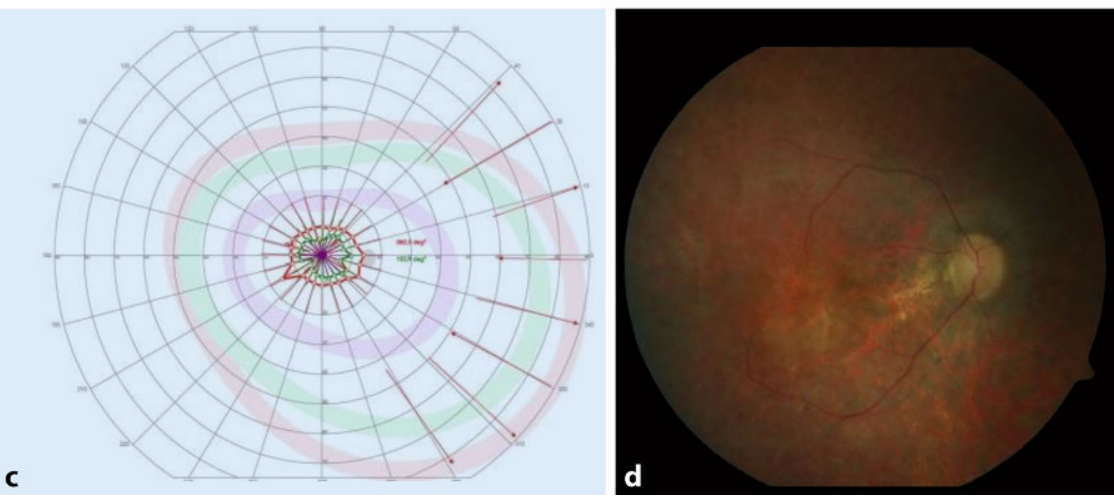

Abb. $2 \Delta$ a Kinetisches Gesichtsfeld (Octopus 900, Fa. Haag-Streit, Wedel) und b Fundusfoto eines gesunden Probanden. c Eingeschränktes Gesichtsfeld und d Fundusfoto mit peripher deutlich atrophen Netzhautarealen

2 erfahrenen Ophthalmologen und einem Statistiker zusammen und berät regelmäßig anhand von Sicherheitskriterien über die Fortsetzung der Studie.

\section{Ziel und Endpunkte der Studie}

Ziel dieser Studie ist es, den Nutzen der TES als Behandlungsmethode bei Retinitis pigmentosa (RP) zu prüfen.

\section{Primärer Endpunkt}

Verminderung des Flächenverlusts im kinetischen Gesichtsfeld (Prüfmarke V4e; gemessen als Differenz zwischen Baseline und 3-Jahres-Visite) des behandelten im Verhältnis zum sham-behandelten $\mathrm{Au}$ ge. Dabei gilt als Ausgangs- bzw. Endwert der Mittelwert von je 2 Gesichtsfeld(GF)Untersuchungen bei der Baseline-Visite bzw. nach 3 Jahren Behandlung.

\section{Sekundäre Endpunkte}

- Kontrastempfindlichkeit,

- bestkorrigierte Sehschärfe (BCVA, „best-corrected visual acuity"),
- statisches Gesichtsfeld,

- Farbsinn,

- Netzhautmorphologie,

- Anzahl und Schweregrad unerwünschter Ereignisse,

- sehbezogene Lebensqualität.

\section{Fazit für die Praxis}

- In einer Multizenterstudie wird die Wirksamkeit der transkornealen Elektrostimulation bei Retinitis pigmentosa (RP) geprüft.

- Über 150 RP-Patienten werden an 18 deutschen Augenkliniken/Praxen 3 Jahre lang unter Therapie mit transkornealer Elektrostimulation (TES; einmal wöchentlich $30 \mathrm{~min}$ ) beobachtet.

- Vor allem das kinetische Gesichtsfeld soll zeigen, ob im Vergleich zum Kontrollauge das behandelte Auge einen deutlich geringeren Gesichtsfeldverlust aufweist.

- Nach Studienauswertung wird der Gemeinsame Bundesausschuss (GBA) den Nutzen der TES-Therapie abschließend bewerten.

\section{Korrespondenzadresse}

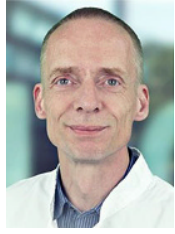

Dr. med. Tobias Peters

Department für Augenheilkunde, Universitätsklinikum Tübingen

Elfriede-Aulhorn-Straße 7, 72076 Tübingen, Deutschland Tobias.Peters@ med.uni-tuebingen.de

Danksagung. Wir danken Prof. Dr. Eberhart Zrenner und der Okuvision $\mathrm{GmbH}$ für ihr großes Engagement welches das Zustandekommen der Erprobungsregelung zur TES-Therapie ermöglicht hat.

Mitglieder der TES-RP-Studiengruppe. Dr. med. Antonios Koutsonas (Universitätsklinikum Aachen, AöR, akoutsonas@ukaachen.de);Prof. Dr.med.Antonia Joussen (Charité - Universitätsmedizin Berlin, studien-augenklinik-cbf@charite.de); Prof. Dr. med. Klaus Rüther (Facharztpraxis für Augenheilkunde); Prof. Dr. med. Frank G. Holz (Universitätsklinikum Bonn, frank.holz@ukb.uni-bonn.de); Prof. Dr. med. Thomas Kohnen (Universitätsklinikum Frankfurt, kohnen@em.uni-frankfurt.de); Dr. med. Fanni Molnar (Universitätsklinikum Freiburg, fanni.molnar@uniklinik-freiburg.de);Prof. Dr. med. Martin Spitzer (Universitätsklinikum Hamburg-Eppendorf, direktor-augenklinik@uke.de); Prof. Dr. med. Klaus Rohrschneider (Universitätsklinikum Heidelberg, klaus.rohrschneider@med.uni-heidelberg.de); Prof. Dr. Johann Roider (Universitätsklinikum Schles wig-Holstein, christoph.ehlken@uksh.de);Prof. Dr. med. Tim Krohne (Uniklinik Köln, tim.krohne@ukkoeln.de); PD Dr. med. Catarina Busch (Universitätsklinikum Leipzig, catharina.busch@medizin.unileipzig.de); PD. Dr. med. Katrin Lorenz (Universitätsmedizin Mainz, katrin.lorenz@unimedizin-mainz.de); Prof. Dr. med. Claudia Priglinger (Augenklinik der Ludwig-Maximilians-Universität München, claudia.priglinger@med.uni-muenchen.de);Prof. Dr. med. Ulrich Kellner (Augenzentrum Siegburg, kellner@augenzentrum-siegburg.de); Prof. Dr. med. Florian Gekeler (Katharinenhospital, f.gekeler@klinikum stuttgart.de); Prof. Dr. med. Peter Szurmann (Knappschaftsklinikum Saar GmbH, augenklinik@kksaar.de); Prof. Dr. med. Katarina Stingl (Universitätsaugenklinik, katarina.stingl@med.uni-tuebingen.de); Prof. Dr. med. Jost Hillenkamp (Universitätsklinikum Würzburg, studien.augenklinik@ukw.de)

\section{Einhaltung ethischer Richtlinien}

Interessenkonflikt. N. Kahle, T. Peters, A. Braun, J. Franklin, C. Michalik, Andreas Stahl für die retina.net e. V., F. Gekeler und B. Wilhelm geben an, dass ihre TES-RP-Studien-bezogenen Leistungen vom G-BA finanziert werden. F. Gekeler gibt Beratertätigkeit bis 2019 für die Retina Implant AG an, deren Produkt die TES bis dahin war, und er war LKP der klinischen Studien $[4,7,8]$. T. Peters und B. Wilhelm übten in den klinischen Studien $[4,7,8]$ bis 2017 im Auftrag der Retina Implant AG CRO-Aufgaben aus. 


\section{Das diagnostische und therapeutische Prinzip}

Alle beschriebenen Untersuchungen am Menschen werden mit Zustimmung der zuständigen EthikKommissionen, im Einklang mit nationalem Recht sowie gemäß der Deklaration von Helsinki von 1975 (in der aktuellen, überarbeiteten Fassung) durchgeführt.

Open Access. Dieser Artikel wird unter der Creative Commons Namensnennung 4.0 International Lizenz veröffentlicht, welche die Nutzung, Vervielfältigung, Bearbeitung, Verbreitung und Wiedergabe in jeglichem Medium und Format erlaubt, sofern Sie den/die ursprünglichen Autor(en) und die Quelle ordnungsgemäß nennen, einen Link zur Creative Commons Lizenz beifügen und angeben, ob Änderungen vorgenommen wurden.

Die in diesem Artikel enthaltenen Bilder und sonstiges Drittmaterial unterliegen ebenfalls der genannten Creative Commons Lizenz, sofern sich aus der Abbildungslegende nichts anderes ergibt. Sofern das betreffende Material nicht unter der genannten Creative Commons Lizenz steht und die betreffende Handlung nicht nach gesetzlichen Vorschriften erlaubt ist, ist für die oben aufgeführten Weiterverwendungen des Materials die Einwilligung des jeweiligen Rechteinhabers einzuholen.

Weitere Details zur Lizenz entnehmen Sie bitte der Lizenzinformation auf http://creativecommons.org/ licenses/by/4.0/deed.de.

\section{Literatur}

1. Bittner AK, Seger K, Salveson R et al (2018) Randomized controlled trial of electro-stimulation therapies to modulate retinal blood flow and visual function in retinitis pigmentosa. Acta Ophthalmol 96:e366-e376

2. Volpe-Waizel DM, Zuche HC, Müller U et al (2020) Metabolic monitoring of transcorneal electrical stimulation in retinitis pigmentosa. Graefes Arch Clin Exp Ophthalmol 258:79-87

3. Ferrari S, Di lorio E, Barbaro V et al (2011) Retinitis pigmentosa: genes and disease mechanisms. Curr Genomics 12:238-249

4. Hartong DT, Berson EL, Dryja TP (2006) Retinitis pigmentosa. Lancet 368:1795-1809

5. Jolly JK, Wagner SK, Martus P et al (2020) Transcorneal electrical stimulation for the treatment of retinitis pigmentosa: a multicenter safety study of the OkuStim(R) system (TESOLA-study). Ophthalmic Res 63:234-243

6. Schatz A, Arango-Gonzalez B, Fischer D et al (2012) Transcorneal electrical stimulation shows neuroprotective effects in retinas of light-exposed rats. Invest Ophthalmol Vis Sci 53:5552-5561

7. Schatz A, Pach J, Gosheva M et al (2017) Transcorneal electrical stimulation for patients with retinitis pigmentosa: a prospective, randomized, shamcontrolled follow-up study over 1 year. Invest Ophthalmol Vis Sci 58:257-269

8. Schatz A, Rock T, Naycheva L et al (2011) Transcorneal electrical stimulation for patients with retinitis pigmentosa: a prospective, randomized, shamcontrolled exploratory study. Invest Ophthalmol Vis Sci 52:4485-4496

9. Sinim Kahraman N, Oner A (2020) Effect of transcorneal electrical stimulation on patients with retinitis pigmentosa. J Ocul Pharmacol Ther 36:609-617

10. Tao Y, Chen T, Liu B et al (2016) The transcorneal electrical stimulation as a novel therapeutic strategy against retinal and optic neuropathy: a review of experimental and clinical trials. Int J Ophthalmol 9:914-919

11. Willmann G, Schäferhoff K, Fischer MD et al (2011) Gene expression profiling of the retina after transcorneal electrical stimulation in wild-type brown Norway rats. Invest Ophthalmol Vis Sci 52:7529-7537

\section{Bilder sagen mehr als Worte}

Wir suchen Ihre informativen und überraschenden Bilder!

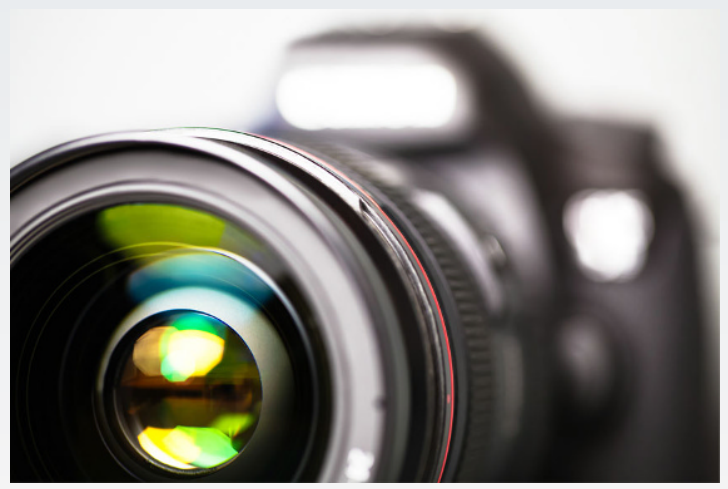

(c) Fotimmz / Fotolia
Verlag und Herausgeberboard von Der Ophthalmologe laden Sie ein, die aufschlussreichsten Bilder aus Ihrem Alltag mit der Community zu teilen.

Schicken Sie uns Ihre Aufahme, erklären Sie uns, wie es zu dem Bild kam und was Sie damit verbinden. Eine Auswahl der informativsten Schnappschüsse werden dann, inklusive der Geschichte dahinter, in Der Ophthalmolloge veröffentlicht. Wir freuen uns auf Ihre Beteiligung!

Senden Sie Ihre Bilder an: Michal Meyer zu Tittingdorf Managing Editor von Der Ophthalmologe michal.meyerzutittingdorf@springer.com 